\title{
OGLE-2009-BLG-092/MOA-2009-BLG-137: A Dramatic Repeating Event With the Second Perturbation Predicted by Real-Time Analysis
}

\author{
Y.-H. Ryu ${ }^{1}$, C. Han ${ }^{1,57,61}$, K.-H. Hwang ${ }^{1}$, R. Street ${ }^{30,59}$, A. Udalski ${ }^{2,56}$, T. Sumi ${ }^{3,55}$, A. Fukui ${ }^{3,55}$, \\ J.-P. Beaulieu ${ }^{4,58}$, A. Gould ${ }^{5,57}$, M. Dominik ${ }^{6,59,60}$ \\ and \\ F. Abe $^{3}$, D.P. Bennett ${ }^{7}$, I.A. Bond ${ }^{12}$, C.S. Botzler ${ }^{8}$, K. Furusawa ${ }^{3}$, F. Hayashi ${ }^{3}$, J.B. Hearnshaw ${ }^{9}$,

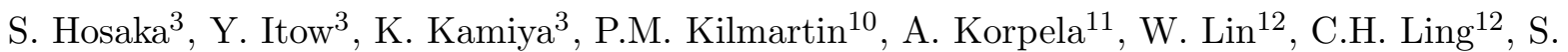 \\ Makita $^{3}$, K. Masuda ${ }^{3}$, Y. Matsubara ${ }^{3}$, N. Miyake ${ }^{3}$, Y. Muraki ${ }^{13}$, K. Nishimoto ${ }^{3}$, K. Ohnishi ${ }^{14}$, \\ Y.C. Perrott $^{8}$, N. Rattenbury ${ }^{8}$, To. Saito ${ }^{15}$, L. Skuljan ${ }^{12}$, D.J. Sullivan ${ }^{11}$, D. Suzuki ${ }^{3}$, W.L. \\ Sweatman $^{12}$, P.J. Tristram ${ }^{10}$, K. Wada ${ }^{13}$, P.C.M. Yock ${ }^{8}$
}

(The MOA Collaboration), M.K. Szymański ${ }^{2}$ M. Kubiak ${ }^{2}$, G. Pietrzyński ${ }^{2,16}$, I. Soszyński ${ }^{2}$, O. Szewczyk ${ }^{16}$, Ł.

Wyrzykowski $^{17}$, K. Ulaczyk ${ }^{2}$

(The OGLE Collaboration)

M. Bos ${ }^{51}$, G.W. Christie ${ }^{18}$, D.L. Depoy ${ }^{19}$, A. Gal-Yam ${ }^{53}$, B.S. Gaudi ${ }^{5}$, S. Kaspi ${ }^{23}$, C.-U. Lee ${ }^{20}$, D. $\mathrm{Maoz}^{23}$, J. McCormick ${ }^{21}$, B. Monard ${ }^{52}$, D. Moorhouse ${ }^{22}$, R.W. Pogge ${ }^{5}$, D. Polishook ${ }^{23}$, Y. Shvartzvald ${ }^{23}$, A. Shporer ${ }^{23}$, G. Thornley ${ }^{22}$, J.C. Yee ${ }^{5}$

(The $\mu$ FUN Collaboration),

M.D. Albrow ${ }^{9}$, V. Batista ${ }^{4,24}$, S. Brillant ${ }^{25}$, A. Cassan ${ }^{4}$, A. Cole ${ }^{27}$, E. Corrales ${ }^{4}$, Ch. Coutures ${ }^{4}$, S. Dieters ${ }^{4,28}$, P. Fouqué2 ${ }^{28}$, J. Greenhill ${ }^{27}$, J. Menzies ${ }^{29}$

(The PLANET Collaboration)

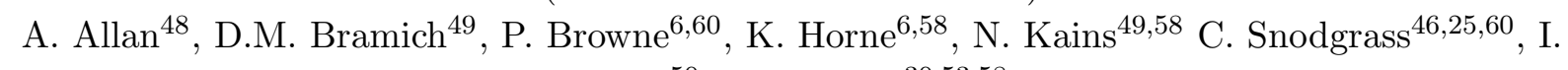
Steele $^{50}$, Y. Tsapras ${ }^{30,53,58}$

(The RoboNet Collaboration) and

V. Bozza ${ }^{31,32,33}$, M.J. Burgdorf ${ }^{34,35}$, S. Calchi Novati $31,32,33$, S. Dreizler ${ }^{36}$, F. Finet ${ }^{37}$, M. Glitrup $^{38}$, F. Grundahl ${ }^{38}$, K. Harpsøe ${ }^{39}$, F.V. Hessman ${ }^{36}$, T.C. Hinse ${ }^{39,40}$, M. Hundertmark ${ }^{36}$ U.G. Jørgensen ${ }^{39,41}$, C. Liebig ${ }^{26,6}$, G. Maier ${ }^{26}$, L. Mancini ${ }^{31,32,33,43}$, M. Mathiasen ${ }^{39}$, S. $\operatorname{Rahvar}^{44,45}$, D. Ricci ${ }^{37}$, G. Scarpetta ${ }^{31,32,33}$, J. Skottfelt ${ }^{39}$, J. Surdej ${ }^{37}$, J. Southworth ${ }^{47}$, J. Wambsganss $^{40}$, F. Zimmer ${ }^{40}$

(The MiNDSTEp Collaboration) 


\footnotetext{
${ }^{1}$ Department of Physics, Institute for Astrophysics, Chungbuk National University, Cheongju 371-763, Korea

${ }^{2}$ Warsaw University Observatory, Al. Ujazdowskie 4, 00-478 Warszawa, Poland

${ }^{3}$ Solar-Terrestrial Environment Laboratory, Nagoya University, Nagoya, 464-8601, Japan

${ }^{4}$ Institut d'Astrophysique de Paris, UMR7095 CNRS-Université Pierre \& Marie Curie, 98 bis boulevard Arago, 75014 Paris, France

${ }^{5}$ Department of Astronomy, Ohio State University, 140 W. 18th Ave., Columbus, OH 43210, USA

${ }^{6}$ School of Physics \& Astronomy, SUPA, University of St. Andrews, North Haugh, St. Andrews, KY16 9SS, UK

${ }^{7}$ Department of Physics, University of Notre Damey, Notre Dame, IN 46556, USA

${ }^{8}$ Department of Physics, University of Auckland, Private Bag 92019, Auckland, New Zealand

${ }^{9}$ University of Canterbury, Department of Physics and Astronomy, Private Bag 4800, Christchurch 8020, New Zealand

${ }^{10}$ Mt. John Observatory, P.O. Box 56, Lake Tekapo 8770, New Zealand

${ }^{11}$ School of Chemical and Physical Sciences, Victoria University, Wellington, New Zealand

${ }^{12}$ Institute of Information and Mathematical Sciences, Massey University, Private Bag 102-904, North Shore Mail Centre, Auckland, New Zealand

${ }^{13}$ Department of Physics, Konan University, Nishiokamoto 8-9-1, Kobe 658-8501, Japan

${ }^{14}$ Nagano National College of Technology, Nagano 381-8550, Japan

${ }^{15}$ Tokyo Metropolitan College of Industrial Technology, Tokyo 116-8523, Japan

${ }^{16}$ Universidad de Concepción, Departamento de Fisica, Casilla 160-C, Concepción, Chile

${ }^{17}$ Institute of Astronomy Cambridge University, Madingley Road, CB3 0HA Cambridge, UK

${ }^{18}$ Auckland Observatory, Auckland, New Zealand

${ }^{19}$ Department of Physics, Texas A\&M University, College Station, TX, USA

${ }^{20}$ Korea Astronomy and Space Science Institute, Daejeon 305-348, Korea

${ }^{21}$ Farm Cove Observatory, Pakuranga, Auckland

${ }^{22}$ Kumeu Observatory, Kumeu, New Zealand

${ }^{23}$ School of Physics and Astronomy, Tel-Aviv University, Tel Aviv 69978, Israel

${ }^{24}$ Institut dÁstrophysique de Paris, UPMC Univ Paris 06, UMR7095, F-75014, Paris, France

${ }^{25}$ European Southern Observatory, Casilla 19001, Vitacura 19, Santiago, Chile

${ }^{26}$ Astronomisches Rechen-Institut, Zentrum für Astronomie der Universitat Heidelberg, Mönchhofstrasse 12-14, 69120 Heidelberg, Germany

${ }^{27}$ School of Math and Physics, University of Tasmania, Private Bag 37, GPO Hobart, Tasmania 7001, Australia

${ }^{28}$ LATT, Université de Toulouse, CNRS, 14 Avenue Edouard Belin, 31400 Toulouse, France

${ }^{29}$ South African Astronomical Observatory, P.O. Box 9 Observatory 7935, South Africa

${ }^{30}$ Las Cumbres Observatory Global Telescope Network, 6740B Cortona Dr, Suite 102, Goleta, CA 93117, USA
} 


\footnotetext{
${ }^{31}$ Università degli Studi di Salerno, Dipartimento di Fisica "E.R. Caianiello", Via Ponte Don Melillo, 84085 Fisciano (SA), Italy

${ }^{32}$ INFN, Gruppo Collegato di Salerno, Sezione di Napoli, Italy

${ }^{33}$ Istituto Internazionale per gli Alti Studi Scientifici (IIASS), Via G. Pellegrino 19, 84019 Vietri sul Mare (SA), Italy

${ }^{34}$ Deutsches SOFIA Institut, Universität Stuttgart, Pfaffenwaldring 31, 70569 Stuttgart, Germany

${ }^{35}$ SOFIA Science Center, NASA Ames Research Center, Mail Stop N211-3, Moffett Field CA 94035, United States of America

${ }^{36}$ Institut für Astrophysik, Georg-August-Universität, Friedrich-Hund-Platz 1, 37077 Göttingen, Germany

${ }^{37}$ Institut d'Astrophysique et de Géophysique, Allée du 6 Août 17, Sart Tilman, Bât. B5c, 4000 Liège, Belgium

${ }^{38}$ Department of Physics \& Astronomy, Aarhus University, Ny Munkegade 120, 8000 Århus C, Denmark

${ }^{39}$ Niels Bohr Institutet, Københavns Universitet, Juliane Maries Vej 30, 2100 København Ø, Denmark

${ }^{40}$ Armagh Observatory, College Hill, Armagh, BT61 9DG, UK

${ }^{41}$ Centre for Star and Planet Formation, Københavns Universitet, Øster Voldgade 5-7, 1350 København Ø, Denmark

${ }^{42}$ Astronomisches Rechen-Institut, Zentrum für Astronomie der Universität Heidelberg (ZAH), Mönchhofstr. 12-14, 69120 Heidelberg, Germany

${ }^{43}$ Dipartimento di Ingegneria, Università del Sannio, Corso Garibaldi 107, 82100 Benevento, Italy

${ }^{44}$ Department of Physics, Sharif University of Technology, P.O. Box 11365-9161, Tehran, Iran

${ }^{45}$ School of Astronomy, IPM (Institute for Studies in Theoretical Physics and Mathematics), P.O. Box 19395-5531, Tehran, Iran

${ }^{46}$ Max Planck Institute for Solar System Research, Max-Planck-Str. 2, 37191 Katlenburg-Lindau, Germany

${ }^{47}$ Astrophysics Group, Keele University, Staffordshire, ST5 5BG, UK

${ }^{48}$ School of Physics, University of Exeter, Stocker Road, Exeter, Devon, EX4 4QL, UK

${ }^{49}$ European Southern Observatory, Karl-Schwarzschild-Straße 2, 85748 Garching bei München, Germany

${ }^{50}$ Astrophysics Research Institute, Liverpool John Moores University, Egerton Wharf, Birkenhead CH41 1LD, UK

${ }^{51}$ Molehill Astronomical Observatory, North Shore, New Zealand

${ }^{52}$ Bronberg Observatory, Pretoria, South Africa

${ }^{53}$ School of Mathematical Sciences, Queen Mary, University of London, London E1 4NS

${ }^{53}$ Benoziyo Center for Astrophysics, the Weizmann Institute, Israel

${ }^{55}$ The MOA Collaboration

${ }^{56}$ The OGLE Collaboration

${ }^{57}$ The $\mu \mathrm{FUN}$ Collaboration

${ }^{58}$ The PLANET Collaboration

${ }^{59}$ The RoboNet Collaboration

${ }^{60}$ MiNDSTEp Collaboration
} 


\begin{abstract}
We report the result of the analysis of a dramatic repeating gravitational microlensing event OGLE-2009-BLG-092/MOA-2009-BLG-137, for which the light curve is characterized by two distinct peaks with perturbations near both peaks. We find that the event is produced by the passage of the source trajectory over the central perturbation regions associated with the individual components of a wide-separation binary. The event is special in the sense that the second perturbation, occurring $\sim 100$ days after the first, was predicted by the real-time analysis conducted after the first peak, demonstrating that real-time modeling can be routinely done for binary and planetary events. With the data obtained from follow-up observations covering the second peak, we are able to uniquely determine the physical parameters of the lens system. We find that the event occurred on a bulge clump giant and it was produced by a binary lens composed of a $\mathrm{K}$ and M-type main-sequence stars. The estimated masses of the binary components are $M_{1}=0.69 \pm 0.11 M_{\odot}$ and $M_{2}=0.36 \pm 0.06 M_{\odot}$, respectively, and they are separated in projection by $r_{\perp}=10.9 \pm 1.3 \mathrm{AU}$. The measured distance to the lens is $D_{\mathrm{L}}=5.6 \pm 0.7 \mathrm{kpc}$. We also detect the orbital motion of the lens system.
\end{abstract}

Subject headings: gravitational lensing

\title{
1. Introduction
}

When a foreground astronomical object is closely aligned to a background star, the light from the background star (source) is amplified by the gravity of the foreground object (lens). The magnification of this gravitational lensing phenomenon depends on the projected separation between the lens and source star. With the change of the separation, the lensing magnification varies in time. For an event caused by a single-mass lens, the brightness variation is characterized by its non-repeating symmetric light curve (Paczyński 1986).

When a star is gravitationally magnified by a lens composed of two masses (binary lens), the resulting light curves become complicated due to the non-linear nature of binary-lensing magnifications (Schneider \& Weiss 1986). The most important feature of binary lensing is caustics, which represent the set of source positions at which the lensing magnification of a point source becomes infinite. Caustics form a single or multiple sets of closed curves each of which is composed of concave curves that meet at cusps. The number, shape, size, and locations of caustic curves vary depending on the separation and mass ratio between the binary components. As a result, light

curves of binary-lensing events exhibit great diversity (Erdl \& Schneider 1993; Mao \& Paczyński 1991).

\footnotetext{
${ }^{61}$ Corresponding author
} 
In current microlensing experiments, events are observed from the combination of survey and follow-up observations. Survey observations, e.g., OGLE (Udalski et al. 2005) and MOA (Sumi et al. 2010), are operated in order to maximize the event rate by monitoring a large area of sky toward the Galactic bulge on a roughly nightly basis using large-format cameras. On the other hand, follow-up observations, e.g., $\mu$ FUN (Gould et al. 2006), PLANET (Beaulieu et al. 2006), and RoboNet (Tsapras et al. 2009), are focused on events alerted by survey observations to densely cover various anomalies including planet-induced perturbations. However, the limited number of telescopes available for follow-up observations restricts the number of events that can be monitored at any given time. For the efficient use of telescopes for follow-up observations, then, it is important to judge which events should be focused upon among the events alerted by survey observations. This judgment can be done based on real-time modeling of events. Real-time modeling is also important to judge the time and duration of follow-up observations. Extended coverage of events is often needed to determine the physical parameters of lenses by measuring subtle deviations caused by long-lasting effects. In addition, planets and binaries often induce multiple perturbations, and resolving additional perturbations is very critical for accurate and precise characterization of lenses. Real-time modeling helps to judge how long and when intensive follow-up observations should be carried out. Despite its importance, routine real-time modeling of binary and planetary lensing events has been difficult due to the large number of parameters to be included in modeling combined with the complexity of $\chi^{2}$ surface of the parameter space. With the development of efficient codes from the efforts of theoretical studies on binary-lensing phenomenology, however, it is now possible to routinely release models of light curves just after or even during the progress of perturbations 1

In this paper, we present results of the analysis of a dramatic repeating event for which the light curve is characterized by two distinct peaks with perturbations near both peaks. The event is highlighted because the second perturbation, which occurred $\sim 100$ days after the first peak, was predicted by the real-time analysis conducted after the first perturbation. With the dense coverage of the second peak by follow-up observations based on the real-time modeling, we are able to measure the physical parameters of the lens.

\section{Observations and Real-Time Modeling}

The event OGLE-2009-BLG-092/MOA-2009-BLG-137 occurred on a Galactic bulge star located at $(\alpha, \delta)_{2000}=\left(17^{\mathrm{h}} 51^{\mathrm{m}} 37^{\mathrm{s}} .95,-29^{\circ} 32^{\prime} 43.23^{\prime \prime}\right)$, which corresponds to the Galactic coordinates $(l, b)=\left(0.15^{\circ},-1.44^{\circ}\right)$. The event was independently detected by the Optical Gravitational Lensing Experiment (OGLE) and Microlensing Observations in Astrophysics (MOA) groups using the $1.3 \mathrm{~m}$ Warsaw telescope of Las Campanas Observatory in Chile and $1.8 \mathrm{~m}$ telescope of Mt. John

\footnotetext{
${ }^{1}$ Since 2009, real-time models of most of anomalous events alerted by survey observations are posted on the webpage http://astroph.cbnu.ac.kr/ cheongho/modelling/model_"year".html, where "year" corresponds to the year that events were discoverd.
} 


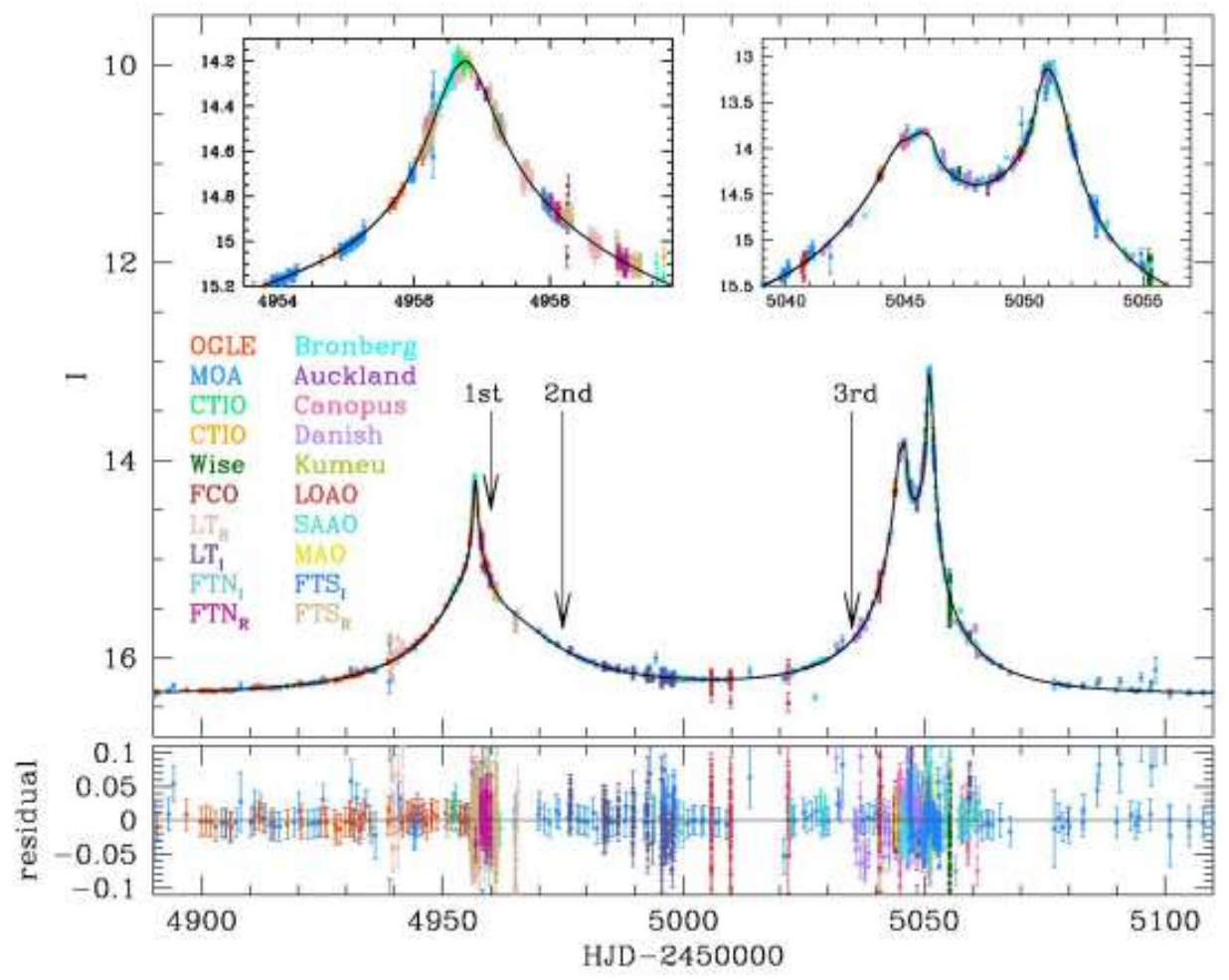

Fig. 1. - Light curve of the microlensing event OGLE-2009-BLG-092/MOA-2009-BLG-137. We note that the MOA data except during the perturbation regions are binned (by 1 day) for clarity. The insets in the upper panel show enlargements of the individual perturbation regions. The lower panel shows the residual from the best-fit model. The arrows represent the times when the real-time analyses were conducted.

Observatory in New Zealand, respectively.

An anomaly alert was issued on on 2009 May 4. Based on the alert, the event was intensively observed by follow-up groups of the Microlensing Follow-Up Network ( $\mu$ FUN), the Probing Lensing Anomalies Network (PLANET), and the RoboNet collaborations. The telescopes used for these follow-up observations include the $\mu$ FUN 1.3 m SMARTS telescope of CTIO in Chile, $\mu$ FUN $0.4 \mathrm{~m}$ of Auckland Observatory, $\mu$ FUN 0.4 m of Farm Cove Observatory (FCO) in New Zealand, $\mu$ FUN $1.0 \mathrm{~m}$ Mt. Lemmon Observatory (LOAO) in Arizona, USA, $\mu$ FUN 0.4 m of Bronberg Observatory in South Africa, RoboNet $2.0 \mathrm{~m}$ Liverpool Telescope (LT) in La Palma, Canary Islands, RoboNet $2.0 \mathrm{~m}$ Faulkes North (FTN) in Hawaii, RoboNet $2.0 \mathrm{~m}$ Faulkes South (FTS) in Australia, and PLANET $1.0 \mathrm{~m}$ of Mt. Canopus Observatory in Australia. Dense coverage by the survey and follow-up observations revealed an anomaly near the peak of the light curve.

Just after the perturbation, real-time analysis of the event was conducted using data available 
at that moment. Modeling showed that the perturbations were produced by a binary lens. The projected separation of the binary system was degenerate between two values, one larger than the Einstein radius and the other smaller. This degeneracy is known as the close/wide binary degeneracy (Dominik 1999b). It was also found that if the perturbation was produced by a wide binary, the source trajectory would pass close to the caustic associated with the other component of the binary, and thus there would be an additional perturbation approximately $\sim 100$ days after the first perturbation.

Another modeling conducted at around HJD $=2454975$ with additionally acquired data resolved the close/wide binary degeneracy and finally predicted the second anomaly. The region between the two peaks was expected to vary smoothly without any major perturbation. In addition, the time gap between the two peaks is too long to be continuously monitored by follow-up observations. Therefore, observations during this period were conducted mostly by survey groups.

A third modeling was conducted at HJD $\sim 2455035$ to precisely predict the time of the second perturbation. From this, the second perturbation was predicted to occur at HJD 2455045. With this prediction, another follow-up campaign was prepared to cover the second perturbation. In addition to the telescopes used for the follow-up observations of the first perturbation, additional telescopes participated in the observations of the second peak. These include the PLANET $1.54 \mathrm{~m}$ Danish Telescope of La Silla Observatory of the Microlensing Network for the Detections of Small Terrestrial Exoplanets (MiNDSTEp) group, $\mu$ FUN $1.0 \mathrm{~m}$ of Wise Observatory in Israel, $\mu$ FUN $0.36 \mathrm{~m}$ of Kumeu Observatory, $\mu$ FUN $0.3 \mathrm{~m}$ of Molehill Astronomy Observatory (MAO) in New Zealand, and PLANET $1.0 \mathrm{~m}$ of SAAO in South Africa. From this campaign, the second peak was also densely resolved.

Figure 1 shows the light curve of OGLE-2009-BLG-092/MOA-2009-BLG-137. In the light curve, the MOA data except during the perturbation regions are binned by 1 day for clarity but the modeling is based on unbinned data. The insets show enlargements of the perturbation regions around the individual peaks. We mark the times when the real-time analyses were conducted.

\section{Modeling}

Due to their great diversity, describing light curves of binary-lensing events requires to include many parameters. The basic structure of light curves of binary-lens events is characterized by six lensing parameters. The first set of three parameters are needed to describe light curves of standard single-lens events: the time required for the source to transit the Einstein radius, $t_{\mathrm{E}}$ (Einstein time scale), the time of the closest lens-source approach, $t_{0}$, and the lens-source separation in units of $\theta_{\mathrm{E}}$ at that time, $u_{0}$ (impact parameter). Describing the deviation caused by the lens binarity requires an additional set of three binary-lensing parameters: the mass ratio between the lens components,

$q$, the projected binary separation in units of the Einstein radius, $s$, and the angle of the source trajectory with respect to the binary axis, $\alpha$. 
In addition to these basic parameters, additional parameters are needed to describe detailed structures of lensing light curves. The event OGLE-2009-BLG-092/MOA-2009-BLG-137 exhibits caustic-induced perturbations at both peaks and thus it is required to consider the finite-source effect to describe deviations occurring when the source approaches and crosses over caustics (Nemiroff \& Wickramasir 1994; Witt \& MaO 1994; Gould 1994). The finite-source effect is parameterized by the ratio of the source radius $\theta_{\star}$ to the Einstein radius $\theta_{\mathrm{E}}$, i.e. $\rho_{\star}=\theta_{\star} / \theta_{\mathrm{E}}$ (normalized source radius).

Due to the large time gap between the two peaks of the light curve, the relative lens-source motion may deviate from a rectilinear one due to the acceleration of the observer's motion induced by the Earth's orbital motion around the Sun (Refsdal 1966; Gould 1992; Smith et al. 2003). We consider this so-called "parallax effect" in the modeling by including the two parallax parameters $\pi_{\mathrm{E}, N}$ and $\pi_{\mathrm{E}, E}$, which are the two components of the microlensing parallax vector $\boldsymbol{\pi}_{\mathrm{E}}$ projected on the sky in the direction of north and east celestial coordinates. The direction of this vector is that of the lens-source relative motion in the frame of the Earth at $t_{0} 2$

We also check the possibility of the change of lens positions caused by its orbital motion. The orbital motion has two effects on lensing magnifications. One causes the binary axis to rotate or, equivalently, makes the source trajectory angle, $\alpha$, change in time. The other effect is causing the separation between the binary components to vary in time (Dominik 1998; Ioka et al. 1999; Albrow et al. 2000). The change in the binary separation alters the shape of the caustic in the course of the event. To the first order, the orbital effect is parameterized by

$$
\alpha(t)=\alpha\left(t_{0}\right)+\omega\left(\frac{t-t_{0}}{t_{\mathrm{E}}}\right)
$$

and

$$
s(t)=s\left(t_{0}\right)+\dot{s}\left(\frac{t-t_{0}}{t_{\mathrm{E}}}\right),
$$

where the orbital lensing parameters $\omega$ and $\dot{s}$ represent the rates of change in the source trajectory angle and the projected binary separation, respectively.

Due to the sheer size of the parameter space, it is difficult to find binary-lensing solutions from brute-force searches. Searches for solutions becomes further hampered by the complexity of the $\chi^{2}$ surface. This complexity implies that even if a solution that seemingly describes an observed light curve is found, it is difficult to be sure that all possible minima have been investigated (Dominik 1999a (b) and thus a simple downhill approach cannot be used. To avoid these difficulties, we use a hybrid approach in whcih grid searches are conducted over the space of a subset of parameters and the remaining parameters are allowed to vary so that the model light curve results in minimum $\chi^{2}$ at each grid point. See also Dong et al. (2006) and Bennett (2010). We choose $s, q$, and $\alpha$ as grid parameters because they are related to the features of light curves in a complicated pattern such that a small change in these parameters can result in dramatic changes in the resulting light

\footnotetext{
${ }^{2}$ We set " $t_{0}$ " as the time of the source star's closest approach to the center of mass of the binary lens.
} 
curves. On the other hand, the other parameters are more directly related to the observed light curve features. We use a Markov Chain Monte Carlo method for $\chi^{2}$ minimization. Once the solutions of the individual grid points are determined, the best-fit model is obtained by comparing the $\chi^{2}$ minima of the individual grid points.

In addition to the difficulties mentioned above, binary-lens modeling suffers from an additional difficulty that arises due to large computations required for modeling. Most binary-lensing events exhibit perturbations induced by caustic crossings or approaches during which the finite-source effect is important. Calculating finite-source magnifications requires a numerical method for which heavy computations are needed. Considering that modeling requires to produce many light curves of trial models, it is important to apply an efficient method for magnification calculations. In our modeling, we use a customized version of the inverse ray-shooting method to calculate finitesource magnifications. In the usual ray-shooting method, a large number of rays are uniformly shot from the image plane, bent according to the lens equation, and land on the source plane. Then, the lensing magnification corresponding to the location of a finite source is computed by comparing the number density of rays on the source surface with the density on the image plane. The main shortcoming of this method is that only a small fraction of rays land on the source surface and most of the rest of the rays are not used for magnification computations. We reduce computation time by minimizing wasted rays. For this, we first make grids on the image plane. We then find the image positions corresponding to the individual positions of the envelope of the source star and then register the grids corresponding to the image positions. We minimize the number of rays by restricting the region of ray shooting only to the registered grids on the image plane. This scheme is similar to that of Rattenbury et al. (2002). We set the width of grids slightly bigger than the source star. If the width is too small, the region inside the image may not be registered. If the width is too big, the fraction of rays not arriving on the source surface will increase. We find that the optimal grid width corresponds to the diameter of the source. This is because lensing-induced distortions always result in images slimmer than the source and thus grids with a width of the source diameter fill the images, thereby minimizing wasted rays. To further speed up the computation, we use the finite-source magnification calculations based on the numerical ray-shooting method only in the region near the caustic, and a simple semi-analytic

hexadecapole approximation (Pejcha \& Hevrovský 2009; Gould 2008) is used in other part of light curves.

\section{Results}

In Table 1, we summarize the results of modeling. We test 5 different models. The first model is based on a static binary lens (no orbital effect) without the parallax effect (standard model). The second model includes the parallax effect. Finally, the orbital motion of the lens is additionally considered in the third model. When the parallax or orbital motion is considered, a pair of solutions resulting from the mirror-image source trajectories with impact parameters $u_{0}>0$ 


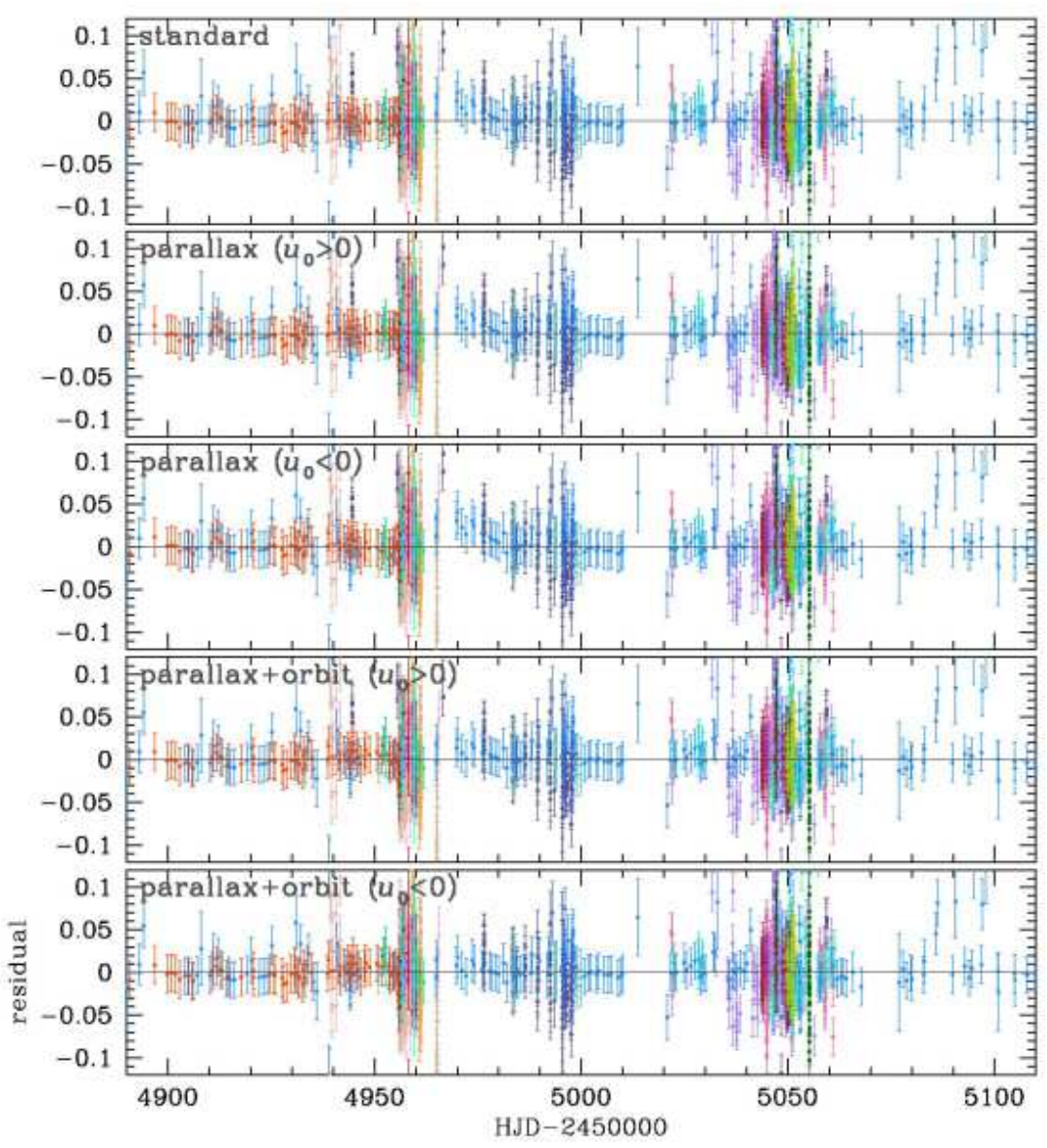

Fig. 2.- Residuals of data from various models.

and $u_{0}<0$ result in slightly different light curves due to the asymmetry of the source trajectories with respect to the binary axis. We, therefore, check both models with $u_{0}>0$ and $u_{0}<0$ whenever the parallax or orbital effect is considered.

From the table, it is found that the model including both the parallax and orbital effects resulting from a source trajectory with $u_{0}<0$ provides the best fit to the observed light curve. It is also found that the parallax effect improves the fit by $\Delta \chi^{2} /$ dof $=40.8 / 6567$, while the orbital effect further improves the fit by $\Delta \chi^{2} /$ dof $=136.0 / 6565$. The differences in the goodness of fit between the solutions can be seen in Figure 2, where we present the residuals of data from the individual models. One finds that the "parallax + orbit" solution removes the systematic residuals that are present in the other solutions. We note that the amount of parallax $\pi_{\mathrm{E}}=\left(\pi_{\mathrm{E}, E}^{2}+\pi_{\mathrm{E}, N}^{2}\right)^{1 / 2}=0.080$ is substantially smaller than the typical values of events for which parallaxes are measured. The orbit-induced changes of the binary separation during the time gap $\Delta t$ between the two peaks of $\Delta s=\dot{s}\left(\Delta t / t_{\mathrm{E}}\right) \sim 0.13$ and the source trajectory angle of $\Delta \alpha=\omega\left(\Delta t / t_{\mathrm{E}}\right) \sim 1.6^{\circ}$ are very small. 


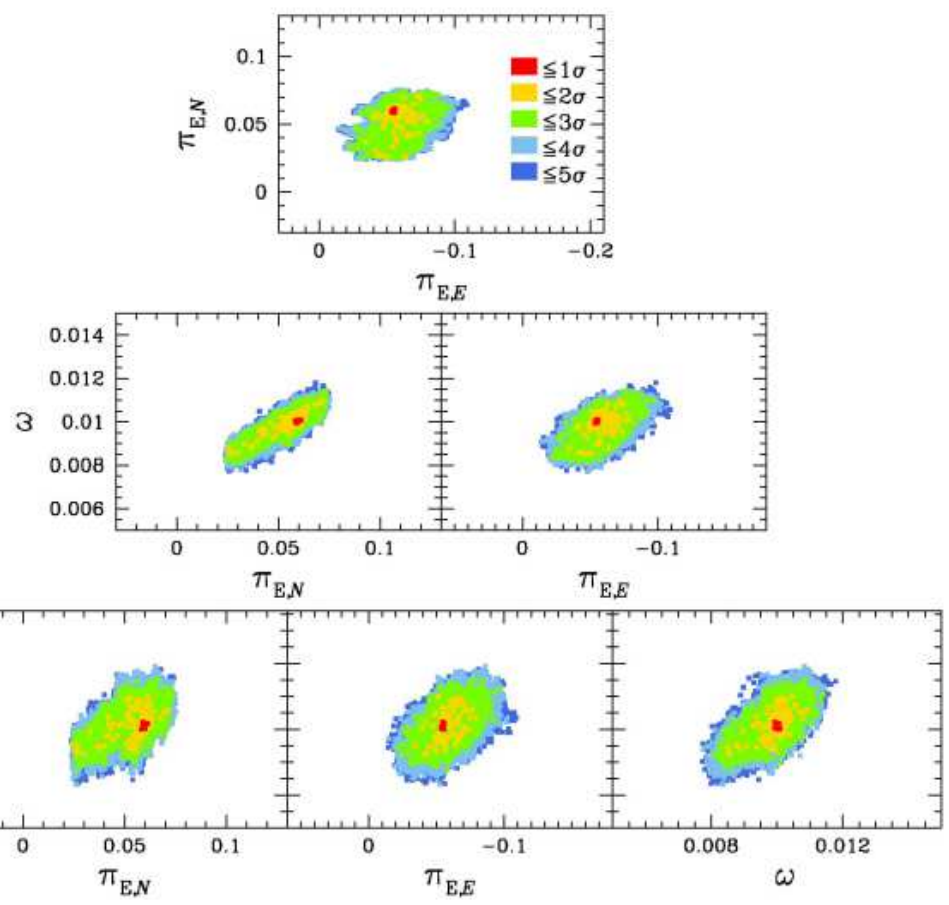

Fig. 3.- Contours of $\chi^{2}$ in the space of parallax and orbital lensing parameters.

Despite their small amplitudes, both parallax and orbital effects are measurable thanks to the dense coverage of the second perturbation from follow-up observations combined with the fact that the event had a long effective time scale and there were two disconnected deviations. Figure 3 presents the contours of $\chi^{2}$ in the space of the combinations of the parallax and orbital lensing parameters. We note that the tangential and radial velocities of the companion relative to the primary are $v_{\mathrm{t}}=r_{\perp} \omega$ and $v_{\mathrm{r}}=r_{\perp}(\dot{s} / s)$, respectively, where $r_{\perp}$ represents the projected binary separation. We, therefore, set the ordinate of the lower panels as $\dot{s} / s$ so that it is in the same order of $\omega$.

In Figure 1, we present the model light curve of the best-fit solution. In Figure 4, we also present the geometry of the lens system based on the best-fit solution. In the figure, the filled circles represent the locations of the lens components, where $M_{1}$ is the heavier component, the two closed curves are the caustics, and the curve with an arrow represents the source trajectory. The two upper panels show enlargements of the regions around the individual caustics. The open circles on the source trajectory represent the source star at the times of observations where the size indicates its finite size and the colors correspond to those of the data points of different observatories adopted in Figure 1. All lengths are normalized by the Einstein radius corresponding to the total mass of the binary.

To determine the physical parameters of the lens system, it is required to measure both the Einstein radius and the lens parallax. The lens parallax is directly measured from modeling. The 


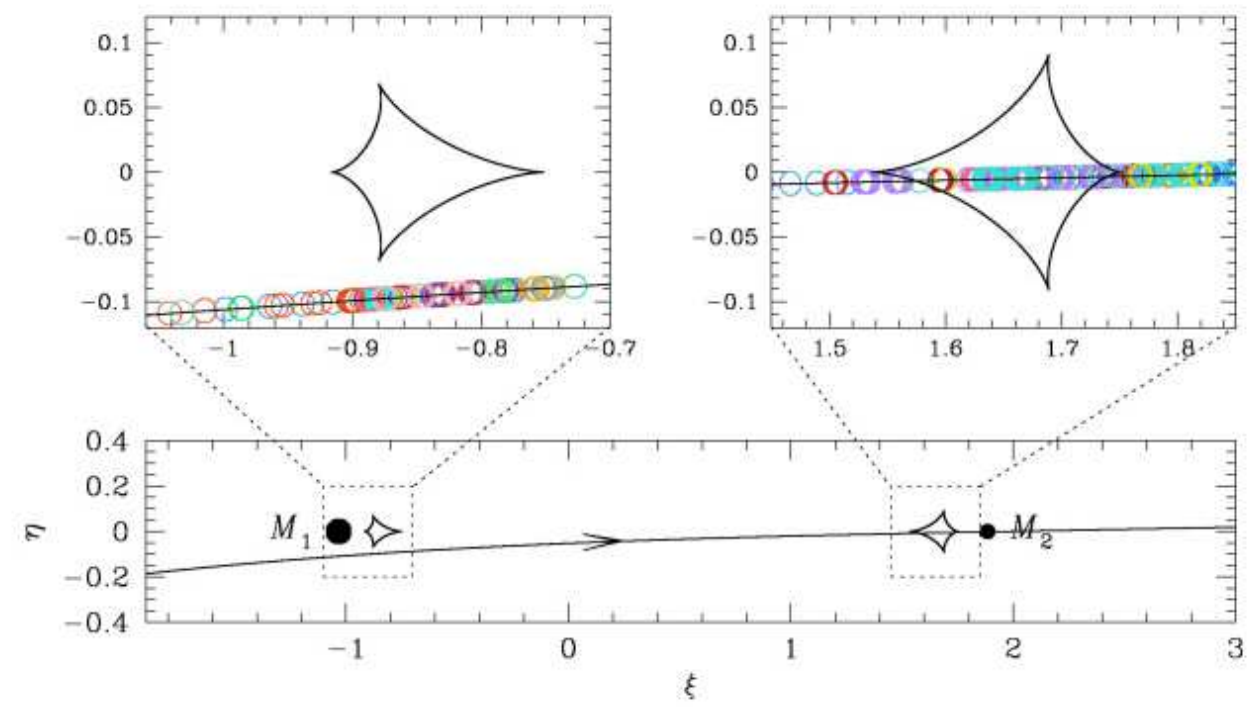

Fig. 4.- Geometry of the lens system responsible for the microlensing event OGLE-2009-BLG092/MOA-2009-BLG-137. The filled circles represent the locations of the lens components, where $M_{1}$ is the more massive component. The two closed curves are the caustics formed by the binary lens. The curve with an arrow represents the source trajectory. The two upper panels show enlargements of the region around the individual caustics, which correspond to the times of the peaks in the light curve at HJD 2454959 and 2455048, respectively. The open circles on the source trajectory represent the source star at the times of observations, where the size indicates its finite size and the colors correspond to those of the data points of different observatories used in Fig. 1, All lengths are normalized by the Einstein radius corresponding to the total mass of the binary.

Einstein radius is measured from the normalized source radius $\rho_{\star}$ combined with the angular size of the source star, $\theta_{\star}$. The angular source size is estimated based on the de-reddened magnitude $I_{0}$ and color $(V-I)_{0}$ of the source star measured from the offset between the source and the centroid of clump giants in the instrumental color-magnitude diagram under the assumption that source and clump giants experience the same amount of extinction (Yoo et al. 2004). In Figure 5, we present the location of the source in the color-magnitude diagram constructed by using the $V$ and $I$ band images taken from CTIO. With the known clump centroid of $\left[(V-I)_{0}, I_{0}\right]_{\mathrm{c}}=(1.04,14.27)$ and the measured offsets of $\Delta(V-I)=(V-I)_{\mathrm{S}}-(V-I)_{\mathrm{c}}=0.123$ and $\Delta I=I_{\mathrm{S}}-I_{\mathrm{c}}=0.429$, the de-reddened color and magnitude of the source star are measured as $\left[(V-I)_{0}, I_{0}\right]_{\mathrm{S}}=(1.16,14.69)$, respectively. Here we adopt the distance to the clump of $8.0 \mathrm{kpc}$ toward the field which is estimated by using the Galactic model of Han \& Gould (2003). Then, the angular source size is determined by first transforming from $(V-I)_{0}$ to $(V-K)_{0}$ using the color-color relation of Bessell \& Brett (1988) and then applying the relation between $(V-K)_{0}$ and the angular stellar radius of Kervella et al. (2004). The determined angular radius of the source star is $\theta_{\star}=6.11 \pm 0.53 \mu$ as, implying that the source star is a Galactic bulge clump giant star. The uncertainty of $\theta_{\star}$ is estimated from the 


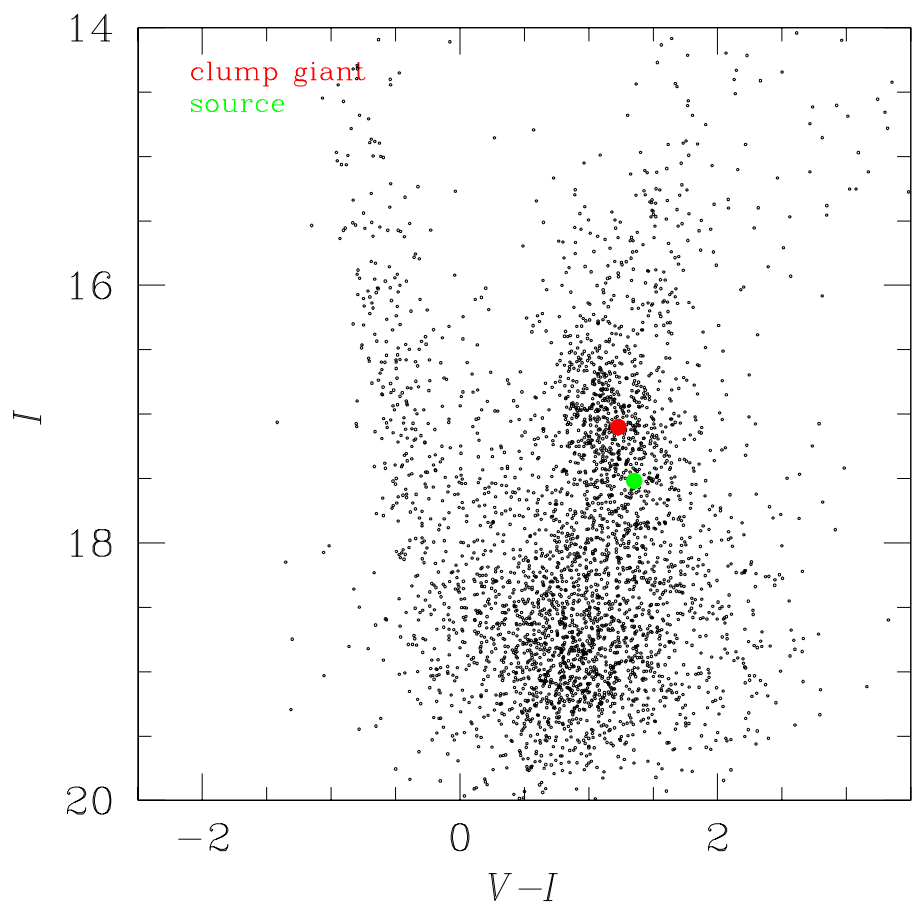

Fig. 5.- Instrumental color-magnitude diagram of stars in the field containing the source star of OGLE-2009-BLG-092/MOA-2009-BLG-137.

combination of those of the colors and magnitudes of the source and the clump centroid and an additional $7 \%$ intrinsic error in the conversion process from the color to the source radius (Yee et al. 2009). Then, the Einstein radius is measured as

$$
\theta_{\mathrm{E}}=\frac{\theta_{\star}}{\rho_{\star}}=0.68 \pm 0.06 \mathrm{mas},
$$

Combining this with the Einstein time scale yields the relative proper motion between the lens and source of

$$
\mu=\frac{\theta_{\mathrm{E}}}{t_{\mathrm{E}}}=7.01 \pm 0.61{\operatorname{mas~} \mathrm{yr}^{-1}}^{-}
$$

With the measured Einstein radius and lens parallax, the mass of the lens and distance to the lens are determined, respectively, as

$$
M=\frac{\theta_{\mathrm{E}}}{\kappa \pi_{\mathrm{E}}}, \quad D_{\mathrm{L}}=\frac{\mathrm{AU}}{\pi_{\mathrm{E}} \theta_{\mathrm{E}}+\pi_{\mathrm{S}}},
$$

where $\kappa=4 G /\left(c^{2} \mathrm{AU}\right), \pi_{\mathrm{S}}=\mathrm{AU} / D_{\mathrm{S}}$ is the parallax of the source, $D_{\mathrm{L}}$ and $D_{\mathrm{S}}$ represent the distances to the lens and source, respectively. For the best-fit model, the determined values are

$$
M=1.04 \pm 0.16 M_{\odot}
$$


and

$$
D_{\mathrm{L}}=5.6 \pm 0.7 \mathrm{kpc}
$$

respectively. From the normalized separation together with the physical Einstein radius $r_{\mathrm{E}}=D_{\mathrm{L}} \theta_{\mathrm{E}}$, the projected separation between the binary companions is estimated as

$$
r_{\perp}=s r_{\mathrm{E}}=10.9 \pm 1.3 \mathrm{AU}
$$

With the known mass ratio, the masses of the individual binary components are estimated, respectively, as

$$
M_{1}=\frac{M}{1+q}=0.69 \pm 0.11 M_{\odot}
$$

and

$$
M_{2}=\frac{q}{1+q} M=0.36 \pm 0.06 M_{\odot} .
$$

Therefore, the event OGLE-2009-BLG-092/MOA-2009-BLG-137 occurred on a bulge clump giant and it was produced by a binary lens composed of a $\mathrm{K}$ and M-type main-sequence stars.

In principle, it is possible to constrain the physical orbital parameters such as the semi-major axis, orbital period, and inclination of the orbital plane from the determined orbital lensing parameters of $\omega$ and $\dot{s}$ (Dong et al. 2009; Bennett et al. 2010). However, we find that this is difficult because the magnitudes of the changes of the binary separation and source trajectory angle are too small. Nevertheless, it is still possible to check the consistency of the orbital parameters using the parameter

$$
\eta=\frac{\left(r_{\perp} / \mathrm{AU}\right)^{3}}{8 \pi^{2}\left(M / M_{\odot}\right)}\left[\omega^{2}+\left(\frac{\dot{s}}{s}\right)^{2}\right]\left(\frac{\mathrm{yr}}{t_{\mathrm{E}}}\right)^{2},
$$

which represents the ratio between kinetic and potential energies. To be a bound system, the parameter should be less than unity. Based on the obtained orbital lensing parameters of the best-fit solution, we find $\eta=0.60$, implying that the result is consistent.

\section{Conclusion}

We analyzed the light curve of a dramatic repeating binary-lens event OGLE-2009-BLG092/MOA-2009-BLG-137 for which the light curve was characterized by two distinct peaks separated by $\sim 100$ days with perturbations near both peaks. By precisely predicting the occurrence of the second perturbation from the analysis of data conducted just after the first perturbation, we demonstrated that real-time modelings can be routinely done for anomalous events. Data covering the second peak obtained from follow-up observations enabled us to uniquely determine the physical parameters of the lens system. From the analysis of the data, we found that the event occurred on a bulge clump giant and it was produced by a binary lens composed of a $\mathrm{K}$ and M-type main-sequence stars. The estimated masses of the individual masses of the binary components were $M_{1}=0.69 \pm 0.11 M_{\odot}$ and $M_{2}=0.36 \pm 0.06 M_{\odot}$, respectively, and they were separated in projection 
by $r_{\perp}=10.9 \pm 1.3 \mathrm{AU}$. The measured distance to the lens was $D_{\mathrm{L}}=5.6 \pm 0.7 \mathrm{kpc}$. Real-time modeling of anomalous lensing events is important not only for efficienct use of observatinal resources but also for precise characterizations of lenses including planetary systems.

Besides that the lens was able to be well characterized by real-time analysis, the event is also important because of its repeating nature. One of the original methods for distinguishing microlensing from variable stars was "non-repeating events". But di Stefano \& Mao (1996) pointed out that this would cause one to miss some binary events and urged that selection should not be done blindly against repeating events. Nevertheless, there have been relatively few repeating binarylens events reported in the literature, e.g. Jaroszyński \& Skowron (2008). Even when they are detected, it is often difficult to distinguish them from those produced by binary source stars. The event OGLE-2009-BLG-092/MOA-2009-BLG-137 results from the special case where the source trajectory passes the central perturbation regions associated with both lens components and thus the binary nature is unambiguously revealed. In current lensing experiments, the majority of binary lenses are detected through the channel of high-magnification events for which the lens binarity can be easily identified from central perturbations (Han \& Hwang 2009). A fraction of these events will result in repeating events although the second peak will not be as dramatic as that of OGLE2009-BLG-092/MOA-2009-BLG-137. A careful analysis of these repeating events can provide an independent way to study the statistics of wide binary stars (Skowron et al. 2009).

We acknowledge the following support: Creative Research Initiative Program (2009-0081561) of National Research Foundation of Korea $(\mathrm{CH})$; Korea Astronomy and Space Science Institute (CUL); NSF AST-0757888 (AG); NASA NNG04GL51G (BSG, AG, RWP); Royal Society University Research Fellow (MD); JSPS20740104 (TS); JSPS19340058 (YM); JSPS20340052 and JSPS18253002 (MOA). The MiNDSTEp monitoring campaign is powered by ARTEMiS (Automated Terrestrial Exoplanet Microlensing Search) (Dominik 2008). Astronomical research at Armagh Observatory is funded by the Department of Culture, Arts and Leisure, Northern Ireland, UK (TCH).

\section{REFERENCES}

Albrow, M. D., et al. 2000, ApJ, 534, 894

Beaulieu, J.-P., et al. 2006, Nature, 439, 437

Bennett, D. P. 2010, ApJ, 716, 1408

Bennett, D. P. 2010, ApJ, 713, 837

Bessell, M. S., \& Brett, J. M. 1988, PASP, 100, 1134

di Stefano, R., \& Mao, S. 1996, ApJ, 457, 93) 
Dominik, M. 1999a, A\&A, 341, 943

Dominik, M. 1999b, A\&A, 349, 108

Dominik, M. 1998, A\&A, 329, 361

Dominik, M., et al. 2008, Astronomische Nachrichten, 329, 248

Dong, S., et al. 2006, ApJ, 642, 842

Dong, S., et al. 2009, ApJ, 695, 970

Erdl, H., \& Schneider, P. 1993, A\&A, 268, 453

Gould, A. 1992, ApJ, 392, 442

Gould, A. 1994, ApJ, 421, L71

Gould, A. 2008, ApJ, 681, 1593

Gould, A., et al. 2006, ApJ, 644, L37

Han, C., \& Hwang K.-H. 2009, ApJ, 707, 1264

Han, C., \& Gould, A. 2003, ApJ, 592, 172

Ioka, K., Nishi, R., \& Kan-ya, Y. 1999, Prog. Theo. Phys., 102, 983

Jaroszyński, M., \& Skowron, J. 2008, Acta Astron., 58, 345

Kervella, P., Thévenin, F., Di Folco, E., \& Ségransan, D. 2004, A\&A, 426, 297

Mao, S., \& Paczyński, B. 1991, ApJ, 374, L37

Nemiroff, R. J., \& Wickramasinghe, W. A. D. T. 1994, ApJ, 424, L21

Paczyński, B. 1986, ApJ, 304, 1

Pejcha, O., \& Heyrovský, B. 2009, ApJ, 690, 1772

Rattenbury, N. J., Bond, I. A., Skuljan, J., \& Yock, P. C. M. 2002, MNRAS, 335, 159

Refsdal, S. 1966, MNRAS, 134, 315

Schneider, P., \& Weiss, A. 1986, A\&A, 164, 237

Skowron, J., Wyrzykowski, Ł., Mao, S., \& Jaroszyński, M. 2009, MNRAS, 393, 999

Smith, M. C., Mao, S., \& Paczyński, B. 2003, MNRAS, 339, 925

Sumi, T., et al. 2010, ApJ, 710, 1641 
Tsapras, Y., et al. 2009, Astronomische Nachrichten, 330, 4

Witt, H. J., \& Mao, S. 1994, ApJ, 429, 66

Udalski, A., et al. 2005, ApJ, 629, 1102

Yee, J. C., et al. 2009, ApJ, 703, 2082

Yoo, J., et al. 2004, ApJ, 603, 139 
Table 1: Fit Parameters

\begin{tabular}{lccccc}
\hline \hline & & \multicolumn{2}{c}{ model } & \multicolumn{2}{c}{ parallax + orbital motion } \\
parameter & standard & $u_{0}>0$ & $u_{0}<0$ & $u_{0}>0$ & $u_{0}<0$ \\
\hline$\chi 2 /$ dof & $6639.03 / 6569$ & $6616.06 / 6567$ & $6598.27 / 6567$ & $6510.61 / 6565$ & $\mathbf{6 5 0 3 . 0 2 / 6 5 6 5}$ \\
$t_{0}(\mathrm{HJD})$ & $4990.441 \pm 0.053$ & $4990.501 \pm 0.047$ & $4990.106 \pm 0.054$ & $4988.473 \pm 0.058$ & $\mathbf{4 9 8 7 . 7 4 4} \pm \mathbf{0 . 0 4 7}$ \\
$u_{0}$ & $-0.062 \pm 0.001$ & $0.060 \pm 0.001$ & $-0.063 \pm 0.001$ & $0.053 \pm 0.001$ & $\mathbf{- 0 . 0 5 1} \pm \mathbf{0 . 0 0 1}$ \\
$t_{\mathrm{E}}($ days $)$ & $35.53 \pm 0.01$ & $35.44 \pm 0.04$ & $35.48 \pm 0.03$ & $35.23 \pm 0.05$ & $\mathbf{3 5 . 2 5} \pm \mathbf{0 . 0 3}$ \\
$s$ & $2.921 \pm 0.001$ & $2.923 \pm 0.001$ & $2.927 \pm 0.001$ & $2.909 \pm 0.002$ & $\mathbf{2 . 8 9 4 6} \pm \mathbf{0 . 0 0 1}$ \\
$q$ & $0.562 \pm 0.001$ & $0.565 \pm 0.001$ & $0.557 \pm 0.002$ & $0.530 \pm 0.001$ & $\mathbf{0 . 5 1 8} \pm \mathbf{0 . 0 0 1}$ \\
$\alpha(\mathrm{rad})$ & $6.2466 \pm 0.0001$ & $0.0343 \pm 0.0003$ & $6.2410 \pm 0.0002$ & $0.0411 \pm 0.0003$ & $\mathbf{6 . 2 4 2 0} \pm \mathbf{0 . 0 0 0 2}$ \\
$\rho_{\star}$ & $0.0101 \pm 0.0001$ & $0.0100 \pm 0.0001$ & $0.0094 \pm 0.0001$ & $0.0091 \pm 0.0001$ & $\mathbf{0 . 0 0 9 0} \pm \mathbf{0 . 0 0 0 1}$ \\
$\pi_{\mathrm{E}, N}$ & - & $-0.055 \pm 0.009$ & $-0.125 \pm 0.005$ & $-0.018 \pm 0.016$ & $\mathbf{0 . 0 5 9} \pm \mathbf{0 . 0 0 8}$ \\
$\pi_{\mathrm{E}, E}$ & - & $-0.019 \pm 0.013$ & $0.057 \pm 0.009$ & $-0.039 \pm 0.013$ & $\mathbf{- 0 . 0 5 4} \pm \mathbf{0 . 0 1 2}$ \\
$\dot{s}\left(t_{\mathrm{E}}^{-1}\right)$ & - & - & - & $0.0315 \pm 0.0016$ & $\mathbf{0 . 0 4 6 4} \pm \mathbf{0 . 0 0 1 3}$ \\
$\omega\left(t_{\mathrm{E}}^{-1}\right)$ & - & - & - & $-0.0068 \pm 0.0007$ & $\mathbf{0 . 0 0 9 9} \pm \mathbf{0 . 0 0 0 6}$ \\
\hline
\end{tabular}

The parameters of the best-fit solution are marked in bold fonts. 